CHEMOMETRIC EVALUATION OF THE INTERNAL REFERENCE MATERIAL (IRM) OF AGRICULTURAL SOILS IN TWO PROVINCIAL MUNICIPALITIES OF LA PAZ

\section{EVALUACIÓN QUIMIOMÉTRICA DEL MATERIAL DE REFERENCIA INTERNO (MRI) DE SUELOS AGRÍCOLAS EN DOS MUNICIPIOS PROVINCIALES DE LA PAZ}

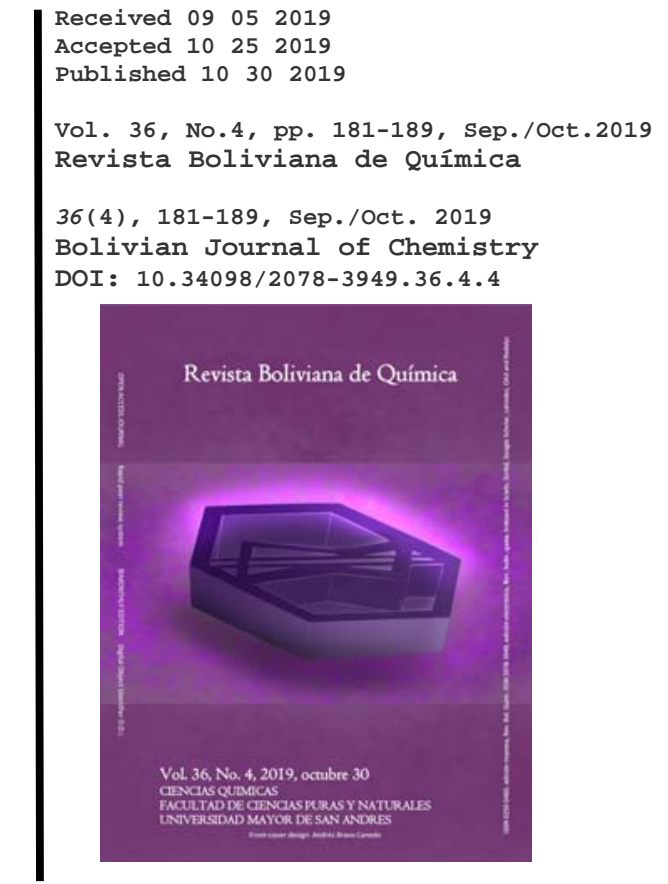

Peer-reviewed

Full original article

Rolando Mamani Quispe ${ }^{1}$, Leonardo Guzmán Alegria ${ }^{1}$, Jorge Chungara Castro², Oswaldo E. Ramos Ramos ${ }^{1}$

${ }^{1}$ Instituto de Investigaciones Químicas (IIQ), Carrera de Ciencias Químicas, Facultad de Ciencias Puras y Naturales FCPN, Universidad Mayor de San Andrés UMSA, P.O. Box 303, Calle Andrés Bello s/n, Ciudad Universitaria Cota Cota, Phone +59122795878, La Paz, Bolivia, www.umsa.bo

${ }^{2}$ Agencia Boliviana de Energía Nuclear ABEN, Centro de Investigación y Aplicaciones Nucleares CIAN, Calle Jaime Mendoza Nro. 987, Esq. Peñaranda, Edificio Torre Soleil (Calacoto), Phone 59122127178, fax 59122129754, http://www.aben.gob.bo/es/contacto, comunicacion@aben.gob.bo

Keywords: Agricultural soils, Natural fertility, Homogeneity, Repeatability, Stability.

Palabras clave: Suelos agrícolas, Fertilidad natural, Homogeneidad, Repetibilidad, Estabilidad.

\title{
ABSTRACT
}

The purpose of the present work is to prepare, characterize and evaluate the physicochemical parameters of two soil samples to be used as internal reference material (MRI). The samples were collected in Yamora (Municipality of Inquisivi) and Viacha (Municipality of Viacha), dried at $100 \mathrm{oC}$, ground, homogenized and quartered. The characterization was carried out by volumetry, potentiometry, conductimetry, UV-Visible spectrophotometry and Atomic Absorption spectrometry.

According to the physical-chemical analysis, the soil of Yamora has a fertility suitable for all types of crops because it has a neutral $\mathrm{pH}$, and high contents in $\mathrm{P}, \% \mathrm{~N}, \% \mathrm{MO}$ and low $\mathrm{CE}$. While the Viacha soil has a low natural fertility due to the strongly alkaline $\mathrm{pH}$, high $\mathrm{Na}+$ contents and low $\mathrm{P}, \% \mathrm{~N}$ and $\% \mathrm{MO}$ contents.

The tests and statistical evaluation of the internal reference materials MRI A (Yamora) and MRI B (Viacha) show homogeneity in all the parameters considered, except the $\mathrm{pH}$ in water. Regarding stability, it was subjected to an evaluation of phosphorus stability assimilable over time and according to statistical results it was shown that the 
stability of MRI B is high, however MRI A is slightly unstable. According to the results, the internal reference materials MRI A (Yamora) and MRI B (Viacha) meet the basic internal reference material requirements for the types of soil studied.

\section{RESUMEN}

El propósito del presente trabajo es preparar, caracterizar y evaluar los parámetros fisicoquímicos de dos muestras de suelo para ser utilizadas como material de referencia interno (MRI). Las muestras fueron colectadas en Yamora (Municipio de Inquisivi) y Viacha (Municipio de Viacha), secadas a $100^{\circ} \mathrm{C}$, molidas, homogenizadas y cuarteadas. La caracterización se efectuó por volumetría, potenciometría, conductimetría, espectrofotometría UV-Visible y espectrometría de Absorción Atómica.

De acuerdo al análisis físicoquímico, el suelo de Yamora presenta una fertilidad apta para todo tipo de cultivo por presentar $\mathrm{pH}$ neutro, y altos contenidos en $\mathrm{P}, \% \mathrm{~N}, \% \mathrm{MO}$ y baja CE. Mientras el suelo de Viacha presenta una baja fertilidad natural debido al $\mathrm{pH}$ fuertemente alcalino, altos contenidos de $\mathrm{Na}^{+}$y bajos contenidos de $\mathrm{P}, \% \mathrm{~N}$ y $\% \mathrm{MO}$.

Las pruebas y evaluación estadística de los materiales de referencia interno MRI A (Yamora) y MRI B (Viacha) muestran homogeneidad en todos los parámetros considerados, excepto el pH en agua. Con relación a la estabilidad, se sometió a una evaluación de estabilidad del fósforo asimilable con el tiempo y según los resultados estadísticos se demostró que la estabilidad de MRI B es alta, en cambio MRI A es levemente inestable. De acuerdo a los resultados, los materiales de referencia interno MRI A (Yamora) y MRI B (Viacha) cumplen los requisitos básicos de material de referencia interna para los tipos de suelo estudiados.

\section{*Corresponding author: rolandogmc1991@gmail.com}

\section{INTRODUCCIÓN}

Los análisis físicos y físicoquímicos en suelos permiten determinar parámetros como: acidez de cambio, disponibilidad de macronutrientes $(\mathrm{P}, \mathrm{K}, \mathrm{Ca}$ y $\mathrm{Mg}$ ), micronutrientes $(\mathrm{Cu}, \mathrm{Fe}, \mathrm{Mn}$ y $\mathrm{Zn}$ ) y otros, como criterio de fertilidad del suelo [1,2].

El análisis de suelo no solo busca cuantificar el contenido total del elemento, sino determinar el contenido asimilable para la planta. Por tanto, el análisis depende en determinar el extractante adecuado para el tipo de suelo y cultivo, así como la calidad y fiabilidad del laboratorio en el que se realicen los análisis.

Para comprobar la fiabilidad de los datos, los laboratorios están usando hoy materiales suficientemente homogéneos y estables con respecto a propiedades específicas, establecidos como aptos para su uso previsto en una medición o en un examen de propiedades cualitativas y cuantitativas. Estos materiales son llamados Material de Referencia Certificado (MRC), que se define como "material de referencia que tiene certificados de uno o varios de sus valores de una o más de sus propiedades, determinados por procedimientos técnicamente válidos llevados a cabo por un organismo competente". La certificación de un organismo competente es lo que hace más confiable al MRC, así como también esto permite determinar la trazabilidad del método [3]. La preparación de un MRC es un proceso delicado donde se deben extremar las precauciones para que el producto cumpla los requisitos que lo harán técnicamente útil y que su uso será el apropiado para los Laboratorios de Ensayo [4].

En este trabajo se prepara y evalúa dos materiales de referencia interno (MRI) realizados en el Centro de Investigación de Aplicaciones Nucleares (CIAN-Viacha) a fin de determinar si es factible su empleo como muestra de control en la evaluación de parámetros fisicoquímicos: $\mathrm{pH}$ en $\mathrm{H}_{2} \mathrm{O}, \mathrm{pH}$ en extracto de $\mathrm{KCl}$, Conductividad Eléctrica (CE), Acidez de cambio, Nitrógeno Total, Materia Orgánica, Fósforo Asimilable y Cationes Intercambiables $\left(\mathrm{Ca}^{2+}, \mathrm{Mg}^{2+}, \mathrm{Na}^{+}, \mathrm{K}^{+}\right)$. Las muestras provienen de dos lugares; la primera, del Municipio de Inquisivi, 1ra. Sección, que se encuentra situada entre las coordenadas: $66^{\circ} 43^{\prime} 29.49^{\prime \prime}$ y $67^{\circ} 17^{\prime} 58$. 62' longitud Oeste, $15^{\circ} 47^{\prime} 34.9^{\prime \prime}$ y $17^{\circ} 18^{\prime} 20.46^{\prime}$ ' latitud Sur y a una altura promedio de 2840 m s.n.m., el área de estudio está ubicada en la comunidad de Yamora. La segunda muestra proviene del municipio de Viacha a $32 \mathrm{~km}$ de la ciudad de

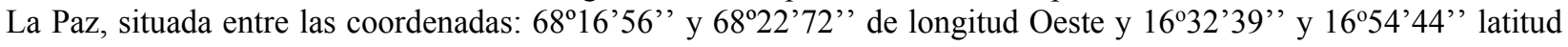
Sur localizada en el Altiplano Norte del departamento de La Paz, con una altitud promedio de 4070 m s.n.m (Figura 1) ubicada en el Centro de Investigaciones y Aplicaciones Nucleares (CIAN). 


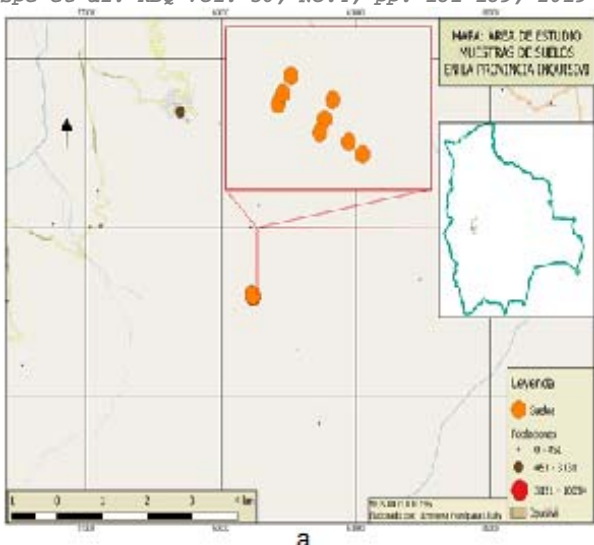

a

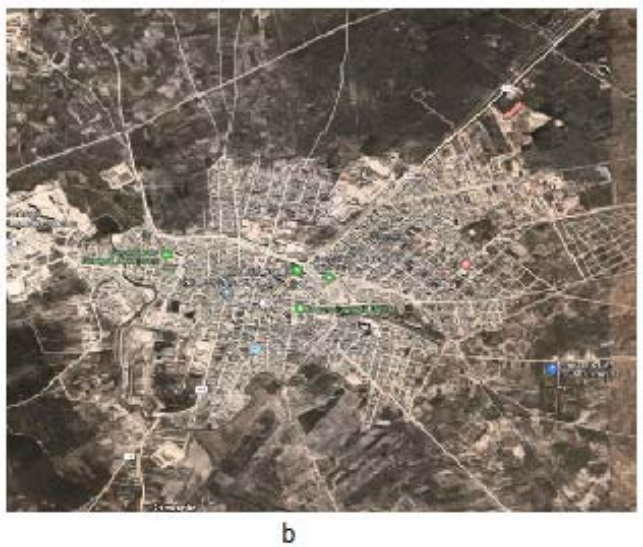

Figura 1. Localización de los puntos de muestreo: a) comunidad de Yamora, Municipio de Inquisivi; b) Centro de Investigaciones y Aplicaciones Nucleares (CIAN-Viacha), Municipio de Viacha.

\section{EXPERIMENTAL}

Todos los reactivos empleados fueron calidad p.a., cloruro de potasio $(\mathrm{KCl})$, fenolftaleína, hidróxido de sodio $(\mathrm{NaOH})$, carbón activado, fluoruro de amonio $\left(\mathrm{NH}_{4} \mathrm{~F}\right)$, molibdato de amonio $\left[\left(\mathrm{NH}_{4}\right)_{6} \mathrm{Mo}_{7} \mathrm{O}_{24}\right]$, cloruro estannoso dihidratado $\left(\mathrm{SnCl}_{2} 2 \mathrm{H}_{2} \mathrm{O}\right)$, fosfato monoácido de sodio $\left(\mathrm{HPO}_{4} \mathrm{Na}_{2}\right)$, bicarbonato de sodio $\left(\mathrm{NaHCO}_{3}\right)$, dicromato de potasio $\left(\mathrm{K}_{2} \mathrm{Cr}_{2} \mathrm{O}_{7}\right)$, ácido sulfúrico $\left(\mathrm{H}_{2} \mathrm{SO}_{4}\right)$, difenilamina $\left[\mathrm{HN}\left(\mathrm{C}_{6} \mathrm{H}_{5}\right)_{2}\right]$, sulfato amónico ferroso $\left(\mathrm{NH}_{4} \mathrm{FeSO}_{4}\right)$, dicromato de potasio $\mathrm{KMnO}_{4}$, hierro reducido, reactivo de selenio, peróxido de hidrógeno $\left(\mathrm{H}_{2} \mathrm{O}_{2}\right.$ al $\left.30 \%\right)$, indicador mixto, acetato de amonio $\left(\mathrm{CH}_{3} \mathrm{COONH}_{4}\right)$ y lantano ( $\left.\mathrm{La}\right)$.

Los equipos empleados fueron: balanza analítica (Sartorius $\pm 0,0005)$, potenciómetro (ThermoScientific con electrodo calomelano), flamómetro (Digital FlameAnalyser, Gallenhamp), espectrofotómetros (UV/visible de doble haz (Helius $\alpha$ ) con celda de cuarzo (1 cm de longitud), espectrómetro de absorción atómica Perkin Elmer Analyst 100), conductimétro OAKTON, agitador magnético (Edmund Buhler a una velocidad de $5 \mathrm{rpm}$, tiempo de reposo de 24 horas para la lectura de $\mathrm{pH}$ ), software estadístico (SPSS, versión 22).

\section{Preparación de la muestra MRI}

La preparación y evaluación de MRI fue aplicada a las dos muestras de la capa arable del suelo, con las siguientes dimensiones: ancho $1 \mathrm{~m}$, largo $1 \mathrm{~m}$ y una profundidad de $20 \mathrm{~cm}$ y tomando una cantidad de $15 \mathrm{~kg}$ de muestra de suelo; se elimina la capa superficial con vegetación. Las muestras fueron secadas a una temperatura de $100^{\circ} \mathrm{C}$ por 24 horas, tamizadas con una malla de $1 \mathrm{~mm}$, para el análisis de parámetros físicos y químicos y sometidos a un proceso de homogenización y resguardadas a temperatura ambiente.

\section{Caracterización y comprobación de homogeneidad}

Se realizó la determinación de: $\mathrm{pH}$ en extracto de $\mathrm{KCl}$, conductividad eléctrica (CE), acidez de cambio, nitrógeno total, materia orgánica, fósforo asimilable y cationes intercambiables $\left(\mathrm{Ca}^{2+}, \mathrm{Mg}^{2+}, \mathrm{Na}^{+}, \mathrm{K}^{+}\right)$. Los resultados fueron tratados estadísticamente para verificar la homogeneidad de las muestras.

\section{Determinación de acidez, alcalinidad y conductividad eléctrica}

La medida de acidez o alcalinidad del suelo $(\mathrm{pH})$ y conductividad fue determinada en extractos de suelo/agua y suelo/solución de $\mathrm{KCl}$ en una relación 1/5 (masa/volumen) utilizando un potenciómetro, y la conductividad eléctrica (CE) utilizando un conductímetro [5].

\section{Acidez de cambio (H-Al):}

Para medir la acidez de suelos, se usa una solución de una sal, no amortiguada, como el cloruro de potasio (KCl). 


\section{Determinación de fósforo asimilable:}

La determinación de fósforo asimilable se realizó con dos métodos de acuerdo al tipo de suelo (ácido o básico). Para suelos ácidos se utilizó el método Bray y Kurtz, en cambio para suelos básicos se utilizó el método de Olsen [7,8].

\section{Determinación de materia orgánica por el método de Walkley y Black:}

El contenido de carbón orgánico (CO) fue determinado por el método de oxidación con dicromato de potasio [9].

\section{Determinación de nitrógeno total por el método Kjeldahl:}

El método consiste en la transformación del nitrógeno de las sustancias nitrogenadas por ebullición con $\mathrm{H}_{2} \mathrm{SO}_{4}$ conc. [10].

\section{Determinación de Cationes intercambiables $\left(\mathrm{Ca}^{2+}, \mathrm{Mg}^{2+}, \mathrm{Na}^{+}, \mathrm{K}^{+}\right)$:}

El método se basa en el desplazamiento de los cationes de calcio y magnesio mediante la solución de acetato de amonio $\left(\mathrm{CH}_{3} \mathrm{COONH}_{4}\right) 1 \mathrm{~N}$. En el filtrado también se determinó $\mathrm{Na}^{+}, \mathrm{K}^{+}$intercambiable [7].

\section{Prueba de homogeneidad y estabilidad:}

Para la prueba de homogeneidad se tomaron dos muestras del cuarteo y se analizó cada parámetro con cinco repeticiones, los datos fueron evaluados mediante el análisis de varianza (ANOVA) para observar diferencias $u$ homogeneidad. La estabilidad se hizo por evaluación de fósforo asimilable con el tiempo en cuatro muestras, el análisis de datos se hizo a través del análisis de varianza de la regresión lineal.

\section{RESULTADOS Y DISCUSIONES}

Los valores de los parámetros determinados (Tabla 1) en el suelo de la comunidad de Yamora presenta: un $\mathrm{pH}$ neutro, $\mathrm{pH}$ en extracto de $\mathrm{KCl}$ moderadamente ácido, la $\mathrm{CE}$ indica un suelo no salino. El valor de acidez de cambio en los suelos de Yamora es $0,0341 \mathrm{mg} \mathrm{H}-\mathrm{Al} / 100 \mathrm{~g}$, el contenido de MO de 3,32\% lo clasifica en la categoría de contenidos moderados. El contenido de $\mathrm{N}$ es $0,31 \%$ y pertenece a la categoría muy alto. El contenido de $\mathrm{Na}^{+}$de 0,13 meq Na/100 g, indica bajo contenido, por el contenido de $\mathrm{K}^{+}$pertenece a la categoría moderado. El contenido de $\mathrm{Ca}^{2+}$ presenta 17,80 meq $\mathrm{Ca}^{2+} / 100 \mathrm{~g}$ y pertenece a la categoría alto. El contenido promedio de $\mathrm{Mg}^{2+}$ de 2,56 meq $\mathrm{Mg}^{2+} / 100 \mathrm{~g}$ y pertenece a la categoría moderado [11]. El valor del contenido de P asimilable es de 258,66 mg P/kg, que lo ubica en la categoría muy alto según la clasificación de Villarroel [11].

De acuerdo a los valores de los parámetros, el suelo de la comunidad Yamora puede clasificarse como apto para todo tipo de agricultura.

Los valores de la tabla 2 (Resultados de las muestras de Viacha, para caracterización y prueba de homogeneidad) muestran que el $\mathrm{pH}$ medido en el extracto acuoso presenta un valor de 8,73; lo que se clasifica como fuertemente alcalino. El pH medido en $\mathrm{KCl}$ es 7,08 y se encuentran dentro de la clasificación neutro, con valores dentro del rango de 6,6-7,0, presentando una $\mathrm{CE}$ de $0,733 \mathrm{mS} / \mathrm{cm}$, y siendo así suelos no salinos con retención de nutrientes y micronutrientes y aptos para la producción agrícola. El valor de acidez de cambio $(\mathrm{H}-\mathrm{Al})$ es 0,0949 mg Al-H /100 g, el contenido de $\mathrm{MO}$ es de $0,63 \%$ y de acuerdo a este valor pertenece a la categoría muy bajo según la clasificación de Villarroel [11]. El contenido de $\mathrm{N}$ es $0,09 \%$ y pertenece a la categoría bajo. Las muestras de Viacha tienen un contenido de $\mathrm{Na}^{+}$de 4,78 meq $\mathrm{Na}^{+} / 100 \mathrm{~g}$ y de acuerdo al contenido de $\mathrm{Na}^{+}$pertenece a la categoría muy alto. El contenido de $\mathrm{K}^{+}$es de 0,45 meq $\mathrm{K}^{+} / 100 \mathrm{~g}$ y de acuerdo al contenido pertenece a la categoría bajo. El contenido de $\mathrm{Ca}^{2+}$ para la muestra de Viacha tiene es de 5,14 meq $\mathrm{Ca}^{2+} / 100 \mathrm{~g}$ y pertenece a la categoría moderado. El contenido de $\mathrm{Mg}^{2+}$ de 3,98 meq $\mathrm{Mg}^{2+} / 100 \mathrm{~g}$ y de acuerdo al contenido pertenece a la categoría moderado. El contenido de $\mathrm{P}$ asimilable es $18,01 \mathrm{mg} / \mathrm{kg}$ y de acuerdo al contenido de P pertenece a la categoría medio según la clasificación de Villarroel [11].

Por los altos contenidos de $\mathrm{Na}^{+}, \mathrm{K}^{+}$y bajos contenidos en $\mathrm{MO}$ y $\mathrm{N}$ este suelo se clasifica como un suelo salino apto solo para determinados cultivos.

Downloadable from: Revista Boliviana 184 de Química. Volumen 36 №4. Año 2019

http://www.bolivianchemistryjournal.org, http://www.scribd.com/bolivianjournalofchemistry 


\section{Prueba de homogeneidad}

Aunque la homogeneidad entre las muestras puede ser evaluadas a través de la prueba t de Student, se ha preferido hacerlo a través del análisis de varianza. En la tabla 3 se presentan los valores de significancia obtenidos para los parámetros fisicoquímicos entre muestras 1 y 2 dentro de cada material de referencia interna.

Las muestras MRI A (Yamora) y MRI B (Viacha) son homogéneas con respecto a los parámetros fisicoquímicos: $\mathrm{pH}$ en $\mathrm{KCl}, \mathrm{CE}, \% \mathrm{MO}, \% \mathrm{~N}$, cationes de cambio $\left(\mathrm{Ca}^{2+}, \mathrm{Mg}^{2+}, \mathrm{Na}^{+}, \mathrm{K}^{+}\right)$y $\mathrm{P}$ asimilable, pero no es homogénea respecto al $\mathrm{pH}$ en agua. Según la evaluación estadística y ANOVA se verifica que las muestras y los resultados obtenidos garantizan que MRI A y MRI B son muestras homogéneas, dado que todos los parámetros cumplen con el criterio excepto por el parámetro $\mathrm{pH}$ en agua de las muestras de Yamora (Inquisivi).

Tabla 1. Resultados de las muestras de comunidad Yamora- provincia Inquisivi para caracterización y prueba de homogeneidad

\begin{tabular}{|c|c|c|c|c|}
\hline \multirow[b]{2}{*}{ Parámetro } & \multirow[b]{2}{*}{ Muestra } & \multirow[b]{2}{*}{ Media } & \multicolumn{2}{|c|}{ Intervalo de confianza al 95\% } \\
\hline & & & Límite inferior & $\begin{array}{l}\text { Límite } \\
\text { superior }\end{array}$ \\
\hline \multirow[b]{2}{*}{$\mathbf{p H}_{\mathrm{H} 2 \mathrm{O}}$} & 1 & 6,74 & 6,72 & 6,76 \\
\hline & 2 & 6,81 & 6,78 & 6,83 \\
\hline \multirow[b]{2}{*}{$\mathbf{p H}_{\mathrm{KCl}}$} & 1 & 5,66 & 5,18 & 6,14 \\
\hline & 2 & 5,82 & 5,50 & 6,14 \\
\hline \multirow{2}{*}{$\begin{array}{l}\mathrm{CE} \\
(\mathrm{mS} / \mathrm{cm})\end{array}$} & 1 & 0,0728 & 0,0691 & 0,0765 \\
\hline & 2 & 0,0708 & 0,0681 & 0,0735 \\
\hline \multirow[b]{2}{*}{ H-Al (mg/100 g) } & 1 & 0,0332 & 0,0324 & 0,0339 \\
\hline & 2 & 0,0350 & 0,0303 & 0,0396 \\
\hline \multirow{2}{*}{$\%$ MO } & 1 & 3,300 & 3,176 & 3,424 \\
\hline & 2 & 3,340 & 3,173 & 3,507 \\
\hline \multirow[b]{2}{*}{$\% \mathbf{N}$} & 1 & 0,297 & 0,259 & 0,334 \\
\hline & 2 & 0,313 & 0,285 & 0,342 \\
\hline \multirow{2}{*}{$\begin{array}{l}\mathrm{Na}^{+} \\
(\mathrm{meq} / 100 \mathrm{~g})\end{array}$} & 1 & 0,1316 & 0,1275 & 0,1357 \\
\hline & 2 & 0,1352 & 0,1270 & 0,1434 \\
\hline \multirow{2}{*}{$\begin{array}{l}\mathrm{K}^{+} \\
(\mathrm{meq} / 100 \mathrm{~g})\end{array}$} & 1 & 0,6748 & 0,6523 & 0,6972 \\
\hline & 2 & 0,6550 & 0,6260 & 0,6839 \\
\hline \multirow{2}{*}{$\begin{array}{l}\mathrm{Ca}^{2+} \\
(\mathrm{meq} / 100 \mathrm{~g})\end{array}$} & 1 & 17,9962 & 17,3830 & 18,6094 \\
\hline & 2 & 17,6230 & 17,1270 & 18,1189 \\
\hline \multirow{2}{*}{$\begin{array}{l}\mathrm{Mg}^{2+} \\
(\mathrm{meq} / 100 \mathrm{~g})\end{array}$} & 1 & 2,5926 & 2,5092 & 2,6759 \\
\hline & 2 & 2,5340 & 2,4501 & 2,6179 \\
\hline \multirow{2}{*}{$\begin{array}{l}\text { Pasimilable, } \\
\text { (mg/kg) }\end{array}$} & 1 & 256,9698 & 244,1352 & 269,8044 \\
\hline & 2 & 263,2392 & 252,8083 & 273,6701 \\
\hline
\end{tabular}

\section{Estabilidad del Material de Referencia Interno (MRI)}

En los dos materiales de referencia interna se realizó, durante tres meses en intervalos de tiempo, la determinación de fósforo asimilable siguiendo el método de Olsen y Bray-Kruzt. La prueba estadística para determinar la estabilidad del fósforo asimilable fue por regresión lineal, debido a que el ANOVA de esta prueba considera una hipótesis nula y alterna sobre la pendiente de la relación del contenido de fósforo con el tiempo, y así a través de su significancia establecer la estabilidad de las muestras, prueba con un nivel de significancia de $\alpha=0,05$. 
Tabla 2. Resultados de las muestras de Viacha, para caracterización y prueba de homogeneidad

\begin{tabular}{|c|c|c|c|c|}
\hline \multirow{2}{*}{ Parámetro } & \multirow{2}{*}{ Muestra } & \multirow{2}{*}{ Media } & \multicolumn{2}{|c|}{$\begin{array}{c}\text { Intervalo de confianza } \\
\text { al } 95 \%\end{array}$} \\
\hline & & & $\begin{array}{l}\text { Límite } \\
\text { inferior }\end{array}$ & $\begin{array}{l}\text { Límite } \\
\text { superior }\end{array}$ \\
\hline \multirow[b]{2}{*}{ рНнго } & 1 & 8,72 & 8,59 & 8,85 \\
\hline & 2 & 8,74 & 8,53 & 8,95 \\
\hline \multirow{2}{*}{$\mathbf{p H}_{\mathrm{KCl}}$} & 1 & 7,14 & 7,11 & 7,16 \\
\hline & 2 & 7,04 & 6,85 & 7,23 \\
\hline \multirow[b]{2}{*}{$\mathrm{CE}(\mathrm{mS} / \mathrm{cm})$} & 1 & 0,7334 & 0,7283 & 0,7384 \\
\hline & 2 & 0,7428 & 0,7167 & 0,7688 \\
\hline \multirow{2}{*}{$\begin{array}{l}\text { H-Al } \quad(\mathrm{mg} / \mathbf{1 0 0} \\
\text { g) }\end{array}$} & 1 & 0,0957 & 0,0832 & 0,1083 \\
\hline & 2 & 0,0942 & 0,0781 & 0,1102 \\
\hline \multirow[b]{2}{*}{ \%MO } & 1 & 0,640 & 0,529 & 0,751 \\
\hline & 2 & 0,640 & 0,529 & 0,751 \\
\hline \multirow[b]{2}{*}{$\% N$} & 1 & 0,094 & 0,075 & 0,113 \\
\hline & 2 & 0,092 & 0,089 & 0,094 \\
\hline \multirow{2}{*}{$\begin{array}{l}\mathrm{Na}^{+} \quad(\mathrm{meq} / \mathbf{1 0 0} \\
\mathrm{g})\end{array}$} & 1 & 4,8612 & 4,4866 & 5,2357 \\
\hline & 2 & 4,7096 & 4,4966 & 4,9225 \\
\hline \multirow{2}{*}{$\begin{array}{l}\mathrm{K}^{+} \\
(\mathrm{meq} / 100 \mathrm{~g})\end{array}$} & 1 & 0,4586 & 0,4182 & 0,4989 \\
\hline & 2 & 0,4456 & 0,4094 & 0,4817 \\
\hline \multirow{2}{*}{$\begin{array}{l}\mathrm{Ca}^{2+} \quad(\mathrm{meq} / 100 \\
\mathrm{g})\end{array}$} & 1 & 5,1258 & 4,9262 & 5,3253 \\
\hline & 2 & 5,15600 & 4,98865 & 5,32335 \\
\hline \multirow{2}{*}{$\begin{array}{l}\mathrm{Mg}^{2+}(\mathrm{meq} / \mathbf{1 0 0} \\
\mathrm{g})\end{array}$} & 1 & 3,9410 & 3,8363 & 4,0456 \\
\hline & 2 & 4,0154 & 3,9749 & 4,0558 \\
\hline \multirow{2}{*}{$\begin{array}{l}\text { Pasimilable, } \\
\text { (mg/kg) }\end{array}$} & 1 & 18,1714 & 16,1225 & 20,2202 \\
\hline & 2 & 17,8506 & 15,5691 & 20,1320 \\
\hline
\end{tabular}

Tabla 3. Valores de significancia (sig.) para la prueba de homogeneidad con ANOVA

\begin{tabular}{|c|c|c|c|c|c|c|c|c|c|c|c|}
\hline \multirow[b]{2}{*}{ MRI } & \multirow[b]{2}{*}{$\mathrm{pH}_{\mathrm{H} 2 \mathrm{O}}$} & \multirow[b]{2}{*}{$\mathrm{pH}_{\mathrm{kCl}}$} & \multicolumn{8}{|c|}{ Valores de significancia (Sig.) } & \multirow[b]{2}{*}{$\mathrm{Mg}^{2+}$} \\
\hline & & & CE & H-Al & $\% \mathbf{N}$ & $\%$ MO & $\mathbf{P}$ & $\mathrm{Na}^{+}$ & $\mathrm{K}^{+}$ & $\mathrm{Ca}^{2+}$ & \\
\hline $\begin{array}{l}\text { A } \\
\text { Yamora }\end{array}$ & 0,0007 & 0,4622 & 0,2566 & 0,2704 & 0,2151 & 0,6075 & 0,3233 & 0,3040 & 0,1728 & 0,2253 & 0,2063 \\
\hline $\begin{array}{l}\text { B } \\
\text { Viacha }\end{array}$ & 0,7927 & 0,1970 & 0,3538 & 0,8347 & 0,6275 & 1,00 & 0,7788 & 0,3572 & 0,5241 & 0,7557 & 0,1029 \\
\hline
\end{tabular}

Criterio de evaluación: $\quad$ Si la Sig. $>0,05$ las muestras son homogéneas respecto al parámetro de estudio.

Si Sig. $<0,05$ las muestras no son homogéneas respecto al parámetro de estudio.

La tabla 4, correspondiente al ANOVA, muestra una significancia de 0,000 para el MRI A, indicando así que existe una relación entre el contenido de $\mathrm{P}$ asimilable y el tiempo, dicho de otra forma, el MRI A Yamora no es estable en el tiempo. Para el MRI B Viacha la significancia es de 0,526 indicando que no existe relación entre el contenido de $\mathrm{P}$ asimilable con el tiempo; esto es, el MRI B es estable en el tiempo (Figura 2).

Tabla 4. Análisis de estabilidad del P asimilable mediante regresión lineal

a) Parámetros de la regresión lineal para el material de referencia interna MRI-A Yamora 
Coeficientes $^{a}$

\begin{tabular}{|c|c|c|c|c|c|c|c|c|}
\hline \multirow{2}{*}{\multicolumn{2}{|c|}{ Modelo }} & \multicolumn{2}{|c|}{$\begin{array}{l}\text { Coeficientes no } \\
\text { estandarizados }\end{array}$} & \multirow{2}{*}{$\begin{array}{c}\begin{array}{c}\text { Coeficientes } \\
\text { estandarizad } \\
\text { os }\end{array} \\
\text { Beta }\end{array}$} & \multirow[b]{2}{*}{ t } & \multirow[b]{2}{*}{ Sig. } & \multicolumn{2}{|c|}{$\begin{array}{c}95.0 \% \text { intervalo de confianza } \\
\text { para B }\end{array}$} \\
\hline & & B & $\begin{array}{l}\text { Error } \\
\text { estándar }\end{array}$ & & & & Limite inferior & $\begin{array}{l}\text { Límite } \\
\text { superior }\end{array}$ \\
\hline \multirow[t]{2}{*}{1} & (Constante) & 194.154 & 4.551 & & 42.657 & .000 & 184.689 & 203.620 \\
\hline & TiempoDias & .468 & .099 & .716 & 4.707 & .000 & .261 & 675 \\
\hline
\end{tabular}

a. Variable dependiente: MRI_A

ANOVA $^{\mathrm{a}}$

\begin{tabular}{|rl|r|r|r|r|l|}
\hline \multicolumn{2}{|c|}{ Modelo } & \multicolumn{1}{c|}{$\begin{array}{c}\text { Suma de } \\
\text { cuadrados }\end{array}$} & gl & $\begin{array}{c}\text { Media } \\
\text { cuadrática }\end{array}$ & F & Sig. \\
\hline 1 & Regresión & 3205.801 & 1 & 3205.801 & 22.152 & $.000^{\text {b }}$ \\
& Residuo & 3039.125 & 21 & 144.720 & & \\
& Total & 6244.927 & 22 & & & \\
\hline
\end{tabular}

a. Variable dependiente: MRI_A

b. Predictores: (Constante), TiempoDias

b) Parámetros de la regresión lineal para el material de referencia interno MRI-B Viacha

\begin{tabular}{|c|c|c|c|c|c|c|c|c|}
\hline \multicolumn{9}{|c|}{ Coeficientes $^{a}$} \\
\hline & & \multicolumn{2}{|c|}{$\begin{array}{l}\text { Coeficientes no } \\
\text { estandarizados }\end{array}$} & \multirow{2}{*}{$\begin{array}{c}\begin{array}{c}\text { Coeficientes } \\
\text { estandarizad } \\
\text { os }\end{array} \\
\text { Beta }\end{array}$} & \multirow[b]{2}{*}{$t$} & \multirow[b]{2}{*}{ Sig. } & \multicolumn{2}{|c|}{$\begin{array}{c}95.0 \% \text { intervalo de confianza } \\
\text { para B }\end{array}$} \\
\hline \multicolumn{2}{|c|}{ Modelo } & B & $\begin{array}{c}\text { Error } \\
\text { estándar }\end{array}$ & & & & Límite inferior & $\begin{array}{l}\text { Límite } \\
\text { superior }\end{array}$ \\
\hline \multirow[t]{2}{*}{1} & (Constante) & 18.551 & .977 & & 18.987 & .000 & 16.513 & 20.589 \\
\hline & TiempoDias & .015 & .023 & .143 & .646 & .526 & -.033 & .062 \\
\hline
\end{tabular}

a. Variable dependiente: MRI_B

ANOVA $^{\text {a }}$

\begin{tabular}{|c|c|c|c|c|c|c|}
\hline & & $\begin{array}{l}\text { Suma de } \\
\text { cuadrados }\end{array}$ & $\mathrm{gl}$ & $\begin{array}{c}\text { Media } \\
\text { cuadrática }\end{array}$ & $\mathrm{F}$ & Sig. \\
\hline \multirow[t]{3}{*}{1} & Regresión & 2.632 & 1 & 2.632 & \multirow[t]{3}{*}{.418} & \multirow[t]{3}{*}{$.526^{\mathrm{b}}$} \\
\hline & Residuo & 126.080 & 20 & \multirow[t]{2}{*}{6.304} & & \\
\hline & Total & 128.712 & 21 & & & \\
\hline
\end{tabular}

a. Variable dependiente: MRI_B

b. Predictores: (Constante), TiempoDias

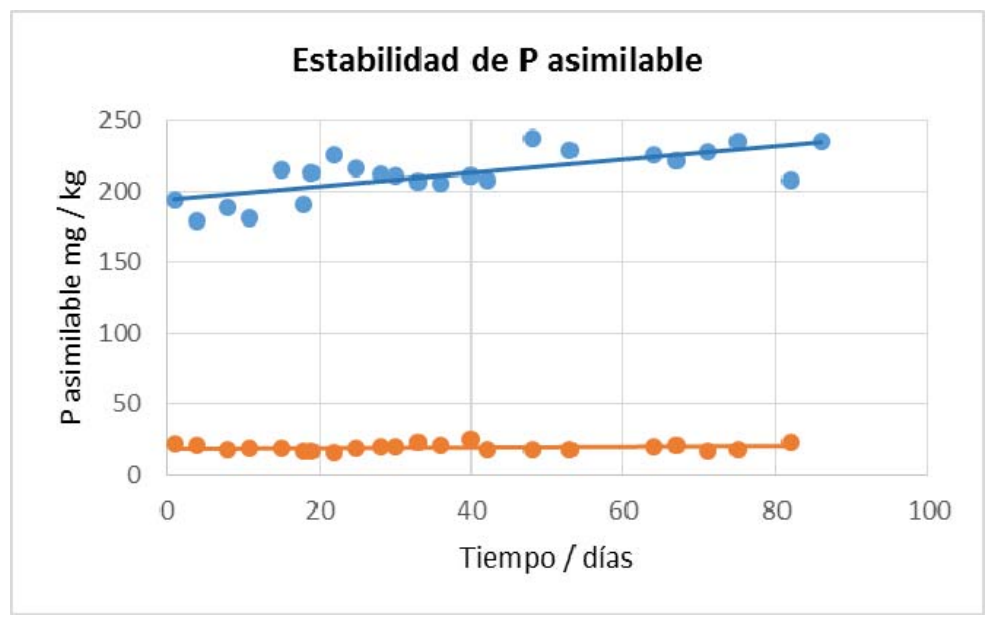

Figura 2. Estabilidad de P asimilable en el tiempo (color azul, MRI A; color naranja MRI B)

\section{CONCLUSIONES}

A partir de los resultados obtenidos de los análisis de las muestras de la capa superficial de suelos se concluye que: 
El MRI A (suelo de la comunidad de Yamora) presenta una textura franco arcillosa; $\mathrm{pH}_{\mathrm{H} 2 \mathrm{O}}$ (valor promedio; 6,8); $\mathrm{pH}_{\mathrm{KCl}}(5,7) ; \mathrm{CE}(0,07173 \mathrm{mS} / \mathrm{cm})$; Acidez de Cambio (0,0341 mg H-Al/100 g); \%N (0,306); \%MO (3,307); P asimilable $(210,663 \mathrm{mg} / \mathrm{kg})$; Cationes intercambiables $(0,133$ meq Na$/ 100 \mathrm{~g} ; 0,665$ meq K$/ 100 \mathrm{~g} ; 17,809$ meq $\mathrm{Ca}^{2+} / 100 \mathrm{~g} ; 2,563$ meq $\left.\mathrm{Mg}^{2+} / 100 \mathrm{~g}\right)$; CIC (21,205 meq/100 g) y TBI (21,171 meq/100 g).

El MRI B [suelo del Viacha, Centro de Investigaciones y Aplicaciones Nucleares (CIAN)] presenta una textura franco arcillosa; $\mathrm{pH}_{\mathrm{H} 2 \mathrm{O}}$ (valor promedio; 8,7$) ; \mathrm{pH}_{\mathrm{KCl}}(7,08) ; \mathrm{CE}(0,733 \mathrm{mS} / \mathrm{cm}) ;$ Acidez de cambio $(0,0949 \mathrm{mg} \mathrm{Al}-\mathrm{H}$ $/ 100 \mathrm{~g}) ; \% \mathrm{~N}(0,0930) ; \% \mathrm{MO}(0,6359) ; \mathrm{P}$ asimilable $(18,011 \mathrm{mg} / \mathrm{kg})$; Cationes intercambiables $\left(4,785 \mathrm{meq} \mathrm{Na}^{+} / 100\right.$ g; 0,452 meq K${ }^{+} / 100$ g; 5,141 meq $\mathrm{Ca}^{2+} / 100$ g; 3,978 meq $\mathrm{Mg}^{2+} / 100$ g); CIC $(14,390$ meq/100 g) y TBI $(14,356$ $\mathrm{meq} / 100 \mathrm{~g})$.

Evaluando los resultados de análisis físicos y químicos como: textura, $\mathrm{pH}, \mathrm{CE}, \mathrm{TBI}, \% \mathrm{MO}, \% \mathrm{~N}$ y $\mathrm{P}$ asimilable se concluye que:

- Los suelos de la comunidad Yamora presenta una fertilidad apta para todo tipo de cultivo.

- Los suelos de CIAN-Viacha presentan una baja fertilidad natural debido a altos contenidos de $\mathrm{Na}^{+}$y bajos contenidos de MO, N y P asimilable, aunque presenta alta capacidad de retención de nutrientes.

- Las muestras MRI A y MRI B son homogéneas con respecto a los parámetros fisicoquímicos: $\mathrm{pH}$ en $\mathrm{KCl}, \mathrm{CE}$, $\% \mathrm{MO}, \% \mathrm{~N}$, cationes de cambio $\left(\mathrm{Ca}^{+2}, \mathrm{Mg}^{+2}, \mathrm{Na}^{+}, \mathrm{K}^{+}\right)$y $\mathrm{P}$ asimilable, pero no es homogénea respecto al $\mathrm{pH}$ en agua. Según la evaluación estadística y ANOVA se verifica que las muestras y los resultados obtenidos se garantiza que MRI A y MRI B son muestras homogéneas, dado que todos los parámetros cumplen con el criterio excepto para el parámetro $\mathrm{pH}$ en agua para las muestras de Yamora (Inquisivi).

En el tema de estabilidad para $\mathrm{P}$ asimilable se concluye que es estable para la muestra de Viacha, mientras que para la muestra de Yamora se observa cierta inestabilidad.

Finalmente, se concluye que las muestras son homogéneas y según los resultados estadísticos obtenidos se establece que MRI A es inestable y MRI B es estable con el tiempo. Por lo tanto, el MRI B puede ser empleado en el control de calidad de los análisis de suelos y puede ser utilizado como Material de Referencia Certificado Interno (MRCI) en cambio MRI A no puede ser usado en los análisis de suelos por su inestabilidad.

\section{RECONOCIMIENTOS}

Al Ing. Rubén Callisaya por la colaboración en el análisis de textura de suelos, a la Tec. Roció Choque por la colaboración en los análisis en el instrumento de Absorción Atómica, a la Lic. Heidi Ruth Mamani Tola por la colaboración en los análisis de muestra en el equipo de Espectrofotómetro (UV/visible), a la Lic. Lizangela Huallpara por la colaboración en la elaboración del mapa del área de estudio, a Damián Torrez y Ing. Gabriela Alba Huanca en la colaboración en los análisis de las muestras de suelos.

\section{REFERENCIAS}

1. Abreu, C.A., Abreu Ferreira, M., Andrade, J.C., Van Raij, B. 1996, Restrictions in the use of correlation coefficients in comparing methods for the determination of the micronutrients in soils, Comunications in Soil Science and Plant Analysis, 29, 1961-1972.

2. Navarro Blaya, S., Navarro García, G. Química agrícola: el suelo y los elementos químicos esenciales para la vida vegetal, Mundi-Prensa, $2^{\text {nd }}$ ed., 2003, México, D.F., México.

3. Anonymous authors. Vocabulario Internacional de Metrología, Conceptos Fundamentales y Generales y Términos Asociados (VIM), Centro Español de Metrología, Ministerio de Industria, Energía y Comercio de España, $3^{\text {ra }}$ ed., 2012, Madrid, España.

4. Anonymous authors. Determinación del contenido de Mercurio en el Material de Referencia MR-CCHEN-001, Albacora, Comisión Chilena de Energía Nuclear, CCHEN, 2000, Santiago, Chile.

5. Karam, A. . Chemical properties of organic soils, Part III, Soil chemical analysis, ed by Y.K. Soon and W.H. Hendershot, In: Soil sampling and methods of analysis, ed by Carter, M.R. \& Gregorich E.G., 2006, CRC, Taylor \& Francis Group, Canadian Society of Soil Science, Boca Raton, Fl, U.S,

6. Colernan, N.T., Weed, S.B., Mccracken, R.J. 1959, Cation exchange capacity and exchangeable cations in Piedemont soils of North Carolina, Soil Sci. Soc. Am. Proc., 23, 146-149.

7. Kaúrichev I. S., Prácticas de edafología. Editorial Mir, 1980, Moscú.

8. Wolf, A.M. Y Baker, D.E. 1965, Comparison of soil test phosphorus by Olsen, Bray P1, Mehlich I and Mehlich III methods. Comm. Soil Sci and Plant Anal, 16, 467-484.

Downloadable from: Revista Boliviana 188 de Química. Volumen 36 №4. Año 2019

http://www.bolivianchemistryjournal.org, http://www.scribd.com/bolivianjournalofchemistry 
Rolando Mamani Quispe et al. RBQ Vol. 36, No.4, pp. 181-189, 2019

9. Cottenie, A., Verloo, M., Kiekens, L., Velghe, G., Camerlynck, R., Chemical analysis of plant and soil laboratory of analytical and agrochemistry, State University Ghent press, 1982, Ghent, Belgium.

10. Vogel, A., Química Analítica Cuantitativa $2^{\text {nd }}$ ed., 1960, Editorial Kapelusz, S.A. Buenos Aires, Argentina.

11. Villarroel J. Manual práctico para la interpretación de análisis de suelos en laboratorio, Edición C. AGRUCO, 1988, Cochabamba, Bolivia. 